\title{
The profile of attention differs between locations orthogonal to and in line with reach direction
}

\author{
Emma E. M. Stewart ${ }^{1}$ - Anna Ma-Wyatt ${ }^{1}$
}

Published online: 7 August 2017

(C) The Psychonomic Society, Inc. 2017

\begin{abstract}
People make movements in a variety of directions when interacting with the world around them. It has been well documented that attention shifts to the goal of an upcoming movement, whether the movement is a saccade or a reach. However, recent evidence suggests that the direction of a movement may influence the spatial spread of attention (Stewart \& Ma-Wyatt, 2015, Journal of Vision, 15(5), 10). We investigated whether the spatiotemporal profile of attention differs depending on where that location is situated relative to the direction of movement, and if this pattern is consistent across different movement effectors. We compared attentional facilitation at locations in line with or orthogonal to the movement, for reach-only, reach-plus-saccade, and saccade-only conditions. Results show that the spatiotemporal profile of attention differs across different movement combinations, and is also different at target locations orthogonal to and in line with the movement direction. Specifically, when a reach alone was made, there was a general decrease in attention at all locations during the movement and a general increase in attention at all locations with a saccade only. However, the concurrent reach and saccade condition showed a premovement attentional facilitation at locations orthogonal to movement direction, but not those in line with the movement direction. These results suggest attentional guidance may be more important at differing time points, depending on the type of movement.
\end{abstract}

Keywords Reaching $\cdot$ Action $\cdot$ Attention $\cdot$ Saccade $\cdot$ Eye movement

Anna Ma-Wyatt

anna.mawyatt@adelaide.edu.au

1 School of Psychology, University of Adelaide, Adelaide, Australia
In a complex environment there are a diverse number of directional hand movements we can make, and as a consequence there are numerous positions for a visual target to be located relative to the direction of any one of these movements. Each of these movements can be accompanied by a shift in visual attention to aid in the guidance of movement planning and execution (Deubel \& Schneider, 1996; Jonikaitis \& Deubel, 2011; Kowler, Anderson, Dosher, \& Blaser, 1995); hence, it is important to understand whether the premotor attentional shift differs at different locations relative to the movement. This study aims to determine how different movement effectors may affect how attention is spread in the immediate vicinity of a movement target. Specifically, whether the spatiotemporal profile of attention differs at different locations relative to movement direction, and whether the movement, or combination of movements being enacted, changes this profile.

The problem of how attention shifts as movements are made to interact with the environment is inherently a spatiotemporal problem. It has been well documented that attention "shifts" to the location of an upcoming movement (Deubel, 2008; Deubel \& Schneider, 1996), and the spatiotemporal profile of this shift provides insight into both the dynamic characteristics and functional benefits of the premotor attentional shift. Attentional facilitation has been observed at the goal of an impending eye movement (Deubel \& Schneider, 1996; Jonikaitis \& Deubel, 2011; Rolfs, Lawrence, \& Carrasco, 2013; White, Rolfs, \& Carrasco, 2013). Kowler et al. (1995) and Deubel and Schneider (1996), using paradigms in which participants were asked to make a sequence of movements, both found perceptual performance on a letterdiscrimination task was better at the location of an upcoming saccade than at other locations. Similarly Zhao, Gersch, Schnitzer, Dosher, and Kowler (2012), Rolfs and Carrasco (2012), Deubel (2008), and Jonikaitis and Deubel (2011) all 
reported a perceptual benefit when a saccade was being planned to the location of the perceptual discrimination task. The majority of studies show that the largest increase in attention is observed around 150-200 ms after cue onset (Castet, Jeanjean, Montagnini, Laugier, \& Masson, 2006; Jonikaitis \& Deubel, 2011; Montagnini \& Castet, 2007), or around $100 \mathrm{~ms}$ before saccade onset (Rolfs \& Carrasco, 2012). The temporal profile of this presaccadic attentional shift is interesting as it demonstrates that attention builds up in the period before the execution of the saccade, suggesting that attending to a location aids in the preparation and planning process of an eye movement.

This premovement attentional shift has also been observed before the onset of goal-directed reaches, with attention increasing at the reach location at approximately $100 \mathrm{~ms}$ after cue onset in one case (Rolfs et al., 2013), or $140 \mathrm{~ms}$ after cue onset in another (Jonikaitis \& Deubel, 2011). This shift is on a similar timescale to that of the presaccadic attentional shift; however, when a reach alone is enacted without an accompanying saccade, the profile of attention can be substantially different. Stewart and Ma-Wyatt (2015) showed that the prereach attentional shift for a reach alone happens far earlier, and the subsequent attentional benefit is far more transient, with a substantial drop in attentional performance as the reach nears endpoint.

The spatial properties of the premovement attentional shift also produce some interesting clues as to how attention is spread around the locus of an upcoming movement. In terms of the premovement attention shift, most studies have examined attentional performance at the planned movement location, compared to a movement-irrelevant location (Deubel \& Schneider, 1996; Kowler et al., 1995). It is worth noting that these studies used paradigms in which the participant was required to make a sequence of movements to target locations that were displayed throughout the task. Attentional performance was enhanced at target locations but not at locations between the targets. While it seems clear that there is an attentional benefit at this target location, the spread and shape of this attentional facilitation is unclear. Some studies suggest that there is a single, focused attentional locus (Song \& Nakayama, 2006), while others argue for a parallel facilitation across multiple future movement locations for both reaches (Baldauf \& Deubel, 2009; Baldauf, Wolf, \& Deubel, 2006) and saccades (Kowler et al., 1995). These studies demonstrate that attention can be allocated to multiple potential movement targets if the task requires it; however, they only probe the spread of attention at specific locations around the movement target, so it is unclear how attention may be allocated at other surrounding locations.

The eye and hand movements that people make to interact with the world must, of course, be made in many different directions relative to the goal location. While there has been little work investigating the deployment of attention relative to the movement direction, some results suggest that it might play a role in modulating the spread of attention. For example, the spatiotemporal profile of attention relative to a reach may be different at locations in line with and orthogonal to reach direction (Stewart \& Ma-Wyatt, 2015). One aspect of saccadic eye movements that has also been shown to have directional perceptual differences is perisaccadic mislocalisation. When making a saccade to a target, the perceived location of the target is often mislocalised, and when a horizontal saccade is being made, the target is mislocalised along this horizontal axis, in the direction of the saccade (Lappe, Awater, \& Krekelberg, 2000), and there is also evidence to indicate that the magnitude of perisaccadic mislocalisation can differ depending on whether a target is presented parallel or orthogonal to a saccade (Kaiser \& Lappe, 2004). While this effect has been shown specifically for saccades, there is evidence to suggest that perisaccadic compression can be observed if participants point to the target, indicating that a common map of space can be accessed for both judgements (Morrone, Ma-Wyatt \& Ross, 2005). These results suggest that the representation of space around the time of the saccade may differ at different locations around a saccade target, and that a similar effect can be observed for reaches. While these studies are not concerned with attention per se, they provide evidence that during a saccade or reach, locations around a target are not represented equally, therefore it is important to determine whether premotor attention also demonstrates such asymmetrical tendencies.

As far as we are aware, there has not yet been any work investigating the effects of saccade or reach direction on the spatial or temporal spread of attention; however, some earlier findings (Stewart \& Ma-Wyatt, 2015) suggest that the direction of a hand movement may affect the shape of the spread of attention around the reach target. This previous study suggests that locations orthogonal to the direction of the hand movement may see a more sustained temporal profile of attention, whereas the locations directly in line with reach direction see a decay in attention over the course of the reach. These results suggest that the particular movement, or combination of movements, may affect how attention is spread around the target, and additionally that there may be some interaction between the type of movement and the location of the target relative to that movement, in determining where attention is allocated. Hence, the question of how the location of a probe relative to a movement may affect attentional facilitation in relation to these different combinations of movements remains unresolved.

Here, we aimed to determine whether the direction of a reach affects the way in which attention facilitates perceptual performance at the locations surrounding the reach target. Thus, the experiment aimed to measure the perceptual 
facilitation at locations in the visual field that were located directly in line with or orthogonal to a reach. As this study also aimed to investigate this attentional profile when different movements were being conducted, the experiment was comprised of a reach-only task, a reach-plus-saccade task, and a saccade-only task.

\section{Method}

\section{Experimental design}

Prior to the main experimental task, a contrast threshold task was conducted for each location, which determined individual observers' contrast thresholds for the stimuli at each target location in the subsequent experimental tasks. The main experimental task comprised three conditions. In the first condition, observers had to reach to a cued location whilst maintaining central fixation. The second condition was identical, except participants had to saccade to the reach endpoint while they made a reach, and in the third condition, participants had to make a saccade alone to the target. For each condition, participants also completed a separate perceptual discrimination task that occurred around the movement target during the movement.

The experiment was a fully repeated-measures design, with all participants completing all three tasks. Participants completed approximately 20 blocks of data for each condition, each of which contained 80 trials. The number of blocks completed varied slightly between participants, as some blocks had to be excluded due to eye-tracking recording errors (e.g. blinks causing loss of pupil image). Participants collected between 19 and 21 blocks of data, for a total of 1,520 to 1,680 trials per participant, for each condition. Blocks for each condition were interleaved.

\section{Participants}

There were four participants. One was an author, whilst three were naïve to the purposes of the experiment. Three were experienced psychophysical observers. All participants had normal or corrected-to-normal vision. Three participants were right-hand dominant, as classified by the Edinburgh handedness test, and used their right hand to point. One participant was classified as left handed and used their left hand to point. As the task involved pointing to one side of the screen only, for the left-handed participant the screen was flipped so they were pointing to the left hand side of the screen with their left hand. Ages ranged from 23 to 29 years. Ethics approval was obtained from the School of Psychology. Participants were free to withdraw from the experiment at any time without penalty.

\section{Equipment}

Stimuli were presented on a 17 -inch ELO touchscreen monitor, with a resolution of $1024 \times 768$ pixels and a screen refresh rate of $75 \mathrm{~Hz}$. The monitor was calibrated to ensure that the monitor's nonlinear gamma function was corrected to be linear. Eye movements were measured using a SR Research EyeLink 1000 eye tracker to record eye position during the task. Eye position was sampled at a rate of $1000 \mathrm{~Hz}$, with a spatial precision of $0.25^{\circ}$, according the manufacturer's specifications. The experiment was run using custom software written in MATLAB using routines from the Psychophysics Toolbox (Brainard, 1997; Pelli, 1997).

\section{Contrast threshold task}

Individual contrast threshold measurements were made for each observer at each of the four probe locations. This ensured that the probe at every location was presented at the observer's contrast threshold level for that specific location, as contrast sensitivity has been shown to change across eccentricities, particularly at levels close to threshold values (Legge \& Kersten, 1987). Separate threshold measurements were taken for the tasks in which there was a concurrent saccade, to ensure the probe was presented at a contrast level that accounted for contrast detection values when the eye had landed at the target. This was particularly important in the reach and saccade condition: as the saccade had generally landed by the time the reach began, the probe was presented at a contrast level that ensured that any change in performance would be due to attentional modulation rather than a change in the preand postsaccadic retinal position of the probe.

For the fixation condition, the participant maintained fixation in the centre of the screen. The target location equivalent to the reach target in the main task was visible. After depressing a key, the probe (and oriented line) appeared at the relevant location being tested. The observer had to indicate the orientation of this probe using the left or right arrow on the keyboard. A QUEST paradigm set to an $82 \%$ threshold (Watson $\&$ Pelli, 1983) adjusted the luminance of the probe. The background luminance of the screen was $27.5 \mathrm{~cd} / \mathrm{m}^{2}$. Forty trials were used to obtain the threshold measurement, and the threshold for each location was tested three times, with the final threshold being the average of the three values (40 trials $\times 3$ blocks for each location). These values were then used such that each probe was presented at this threshold value for each participant.

A similar paradigm was used to measure contrast thresholds when a saccade was being made. As described above, the observer depressed a key, after which a beep occurred to signal the observer to saccade to the saccade target on the screen. The perceptual probe appeared $300 \mathrm{~ms}$ after the beep in order to account for variable saccade latencies and to avoid any 
effects of saccadic suppression. Upon completion of the saccade, the observer had to indicate the orientation of the line, and a QUEST paradigm was used, as described above. This ensured that the probe would appear at a contrast level that was at the threshold for that approximate retinal location during a saccade.

\section{Reach-only task}

At the start of each trial, a grey fixation circle appeared in the middle of the screen that was $0.25^{\circ}$ in size and $18 \%$ Michelson contrast from the background. Two saccade targets appeared on the screen: one was to the right of the fixation point, at $10^{\circ}$ eccentricity; the other was $10^{\circ}$ above the fixation point. The targets were circles $0.75^{\circ}$ in diameter and $10 \%$ contrast from the background. Probes were placed in line with the reach (that is, between the initial start location and the target location) or orthogonal to the reach direction (see diagram in Fig. 1).

As shown in Fig. 1b, the reach trial started when the participant touched the central fixation point on the screen. After a random delay of 5 to $1,000 \mathrm{~ms}$ (calculated at 5-ms intervals on a trial-by-trial basis using MATLAB's randperm function), a cue appeared to signal whether a reach had to be made to the location at the right or at the top of the screen. This cue was had $100 \%$ validity for the movement target location and also indicated the general location of the perceptual probe (above fixation or to the right of fixation).

The cue was a white line of $1.5^{\circ}$ size and $17 \%$ contrast, which pointed either rightwards or upwards. The cue onset also signalled the start of the SOA, after which the perceptual probe would appear at one of two locations next to the reach target. SOA lengths were 13, 107, 200, 306, or $400 \mathrm{~ms}$. The probe was a white line of $0.5^{\circ}$ length, oriented at and an angle of $45^{\circ}$ left or right. The probe was presented at an individually determined contrast level for the participant, condition, and location.

As shown in Fig. 1a, parallel probe locations were located $8^{\circ}$ from the fixation point, between the fixation and target, either to the right of the fixation point (P1) or directly above fixation (P2). Orthogonal probe location O1 was located $2^{\circ}$ above the reach target, and location $\mathrm{O} 2$ was located at $2^{\circ}$ to the left of the reach target, such that both probes appeared at $90^{\circ}$ to the direction of the reach. Locations were chosen to be consistent with locations tested in Stewart and Ma-Wyatt (2015). In this previous study, multiple locations were tested surrounding a movement target, at different eccentricities. As the pattern of results was extremely consistent for eccentricities at one given direction relative to the target, this study just tested one eccentricity at the locations relative to the target.

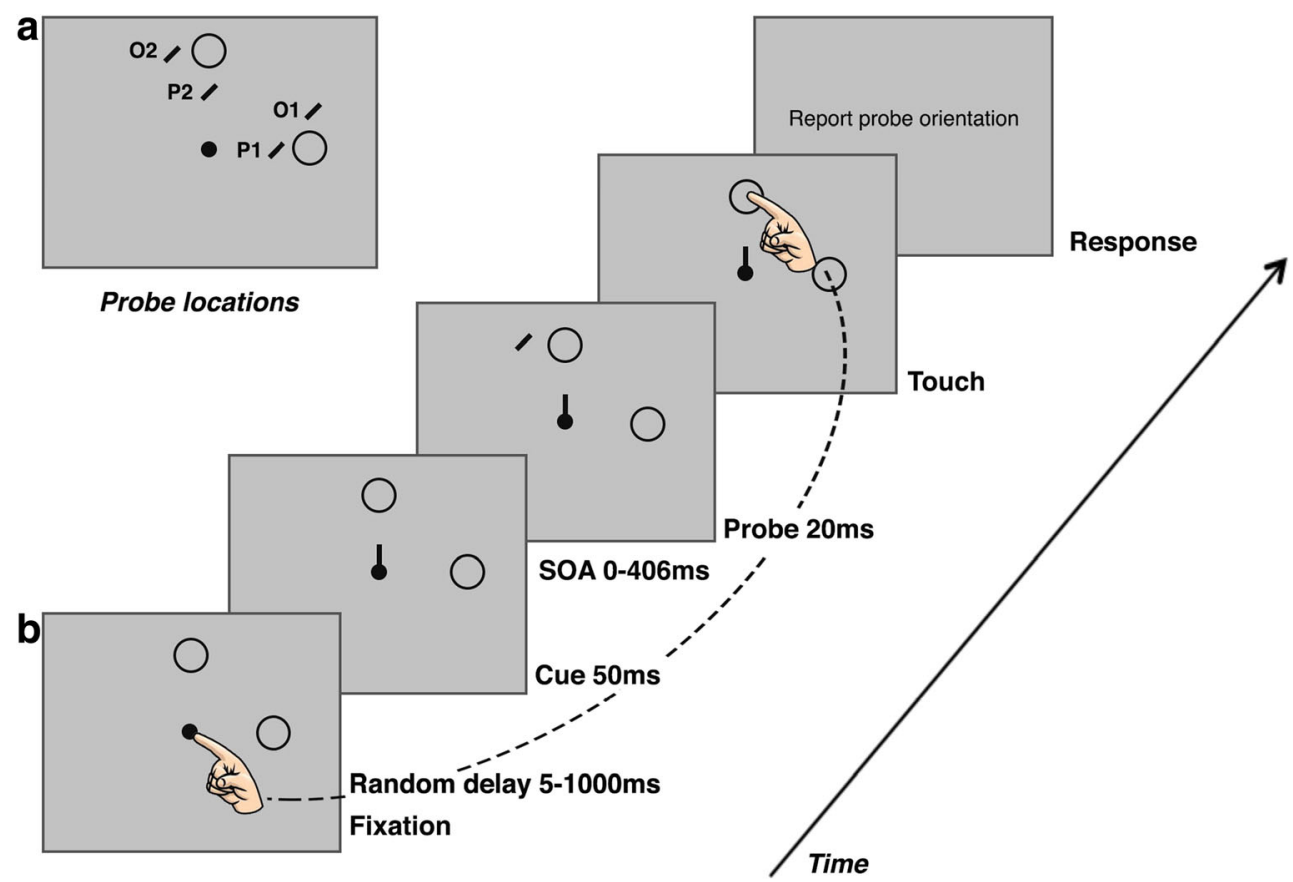

Fig. 1 a Potential probe locations. Location P1 is $8^{\circ}$ right from the fixation point. Location $\mathrm{O} 1$ is $10^{\circ}$ right from the fixation point and $2^{\circ}$ above the right touch target. Location P2 is $8^{\circ}$ above the fixation point. Location $\mathrm{O} 2$ is $10^{\circ}$ above fixation point and $2^{\circ}$ to the left of the upper touch target. b Timeline of events in a trial. Participants fixated a central fixation point and touched this point to start a trial. After a variable delay, a cue indicated which target to reach to, and signalled the start of the movement. During the movement, at a variable SOA, the perceptual probe appeared. For reaching conditions, auditory negative feedback was given if the reach time was slower than $600 \mathrm{~ms}$. After the movement was completed, participants reported probe orientation 
Participants were instructed to maintain central fixation at all times. Cues occurred with equal probability to each movement location.

\section{Reach and saccade task}

The experimental paradigm was identical to the reach-only task, as described above (see Fig. 1), with the addition of a concurrent saccade to the target location.

\section{Saccade-only task}

The experimental paradigm was comparable to the reaching tasks, except a saccade alone was completed, with no concurrent reach. The only other difference was that to start a trial, participants depressed the enter key on the keyboard, and on the 'go' signal, made a saccade to the target.

\section{Results}

\section{Data exclusions}

For the saccade-plus-reach condition, saccades were analysed and trials were excluded when the saccade was not to the target, where there was a blink, or where the eye tracker dropped the eye trace. Additionally, trials in which the saccade latency was less than $100 \mathrm{~ms}$ were excluded to avoid anticipatory saccades (He \& Kowler, 1989). Trials were also excluded where the probe appeared during a window from -50 to $+25 \mathrm{~ms}$ relative to saccade onset, when saccadic suppression may have occurred $(50 \mathrm{~ms}$ before the saccade was initiated until $25 \mathrm{~ms}$ into the saccade; Morrone, Ross, \& Burr, 2005). Reaches were excluded where the reach latency, reach time, and reach accuracy were above \pm 2 standard deviations from the mean. For the reach-only condition, $89 \%$ of trials were included, comprising a total of 5,274 trials. For the saccade-only condition, $76 \%$ of trials were included, comprising a total of 4,831 trials. For the saccade and reach condition, $77 \%$ of trials were included, comprising a total of 4,335 trials. The proportion of trials excluded differs between conditions due to the differing exclusion criteria: for example, in the saccade-plus-reach condition, the exclusion rate is the highest as exclusions were made on the basis of both saccade and reach dynamics and accuracy. Exclusions ensured that the observed performance was due only to the facilitation from premotor attention, and that confounds such as saccadic suppression and retinal location of the attentional probe did not affect results.

\section{Movement dynamics}

Saccades were classified using custom-written software as movements that exceeded velocity of $80 \mathrm{deg} / \mathrm{s}$ and acceleration of 9,500 deg/s. Saccade latency was measured as the time between the movement cue and the start of the saccade. Hand latency was measured as the time between the movement cue and the time the finger left the centre of the screen. Eye-hand latency was measured as the time between saccade onset and reach onset. Movement dynamics were consistent across participants, as represented by stacked density plots in Fig. 2. For the reach-only condition, median reach latency was $287 \mathrm{~ms}$, interquartile range (IQR): $66 \mathrm{~ms}$. For the saccade-only condition, the median saccade latency was $178 \mathrm{~ms}$, IQR: $31 \mathrm{~ms}$. For the saccade and reach condition, the median reach latency was $286 \mathrm{~ms}$, IQR: $61 \mathrm{~ms}$, and the median saccade latency was 174 ms, IQR: 26 ms. Median eye-hand latency was $111 \mathrm{~ms}$, IQR: $52 \mathrm{~ms}$. Note that medians and interquartile ranges are reported due to the nonnormal nature of movement distributions.

\section{Performance relative to SOA}

Performance at each location was measured at a time point relative to the cue onset. Figure 3 shows this performance for each condition. For the locations that were in line with the movement (P1 and P2), it can be seen that in the reachonly condition, peak performance occurs at the shortest SOA, with performance degrading by about $30 \%$ throughout the preparation and execution of the reach. Conversely, for the reach and saccade condition at these locations, performance remains fairly consistently low across all SOAs. For the locations orthogonal to the direction of movement (O1 and $\mathrm{O} 2$ ), performance on the reach-only condition is similar to locations P1 and P2, except the drop in performance is an approximately $10 \%-15 \%$ difference. For the reach and saccade condition, however, performance is lowest at the shortest SOA and builds up by about 30\%, with the peak after the $200 \mathrm{~ms}$ SOA.

This figure also shows that for each condition, reaching performance is generally better during different parts of the movement preparation. For example, peak performance for the reach-only condition generally occurs at an earlier SOA (see Fig. 3a), whereas peak performance in the reach and saccade condition occurs at different points, depending on probe location (see Fig. 3c). This difference in performance across the time course was quantified by comparing the difference between the first SOA and the last SOA across participants for each movement condition (see Fig. 3d). This allows a visualization and comparison of the overall trend in the increase/decrease in attention across different movements.

Linear mixed models were used to analyse the influence of SOA, location, and probe location relative to a 


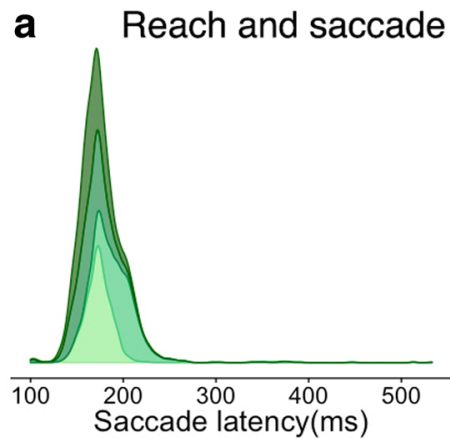

\section{b}

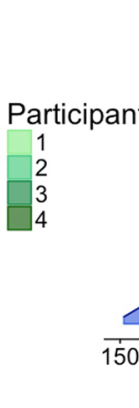

th

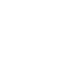

$-\lambda$

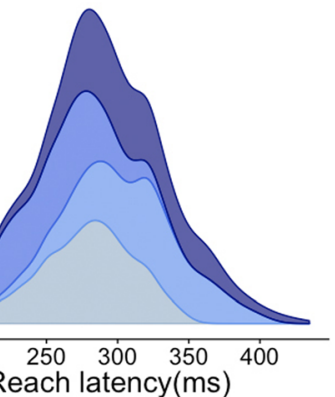

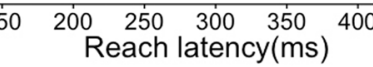

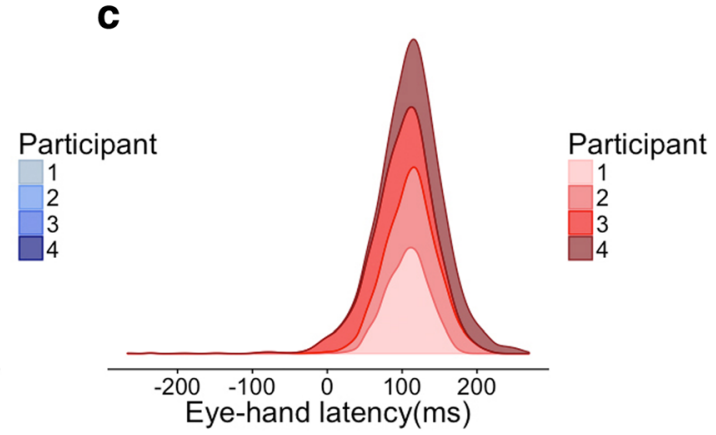

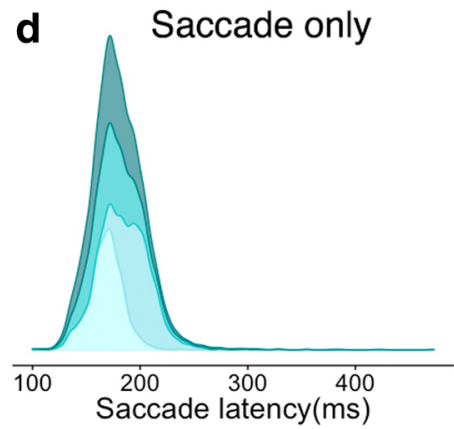

Fig. 2 Movement dynamics for each participant represented as stacked density plots. a saccade latencies for the saccade-plus-reach condition. $\mathbf{b}$ Reach latencies for the saccade-plus-reach condition. c Eye-hand
Reach only

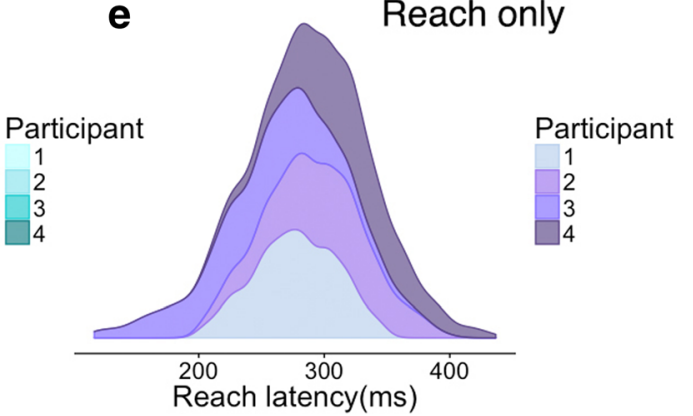

latencies for the saccade-plus-reach condition. d Saccade latencies for the saccade-only condition. e Reach latencies for the reach-only condition movement on performance (fixed effects: SOA, location, probe location; random effect: participant). Mean scores for each participant at each SOA and location were used for these calculations.

For the reach-only condition, there was a significant effect of the following on performance: SOA: $F(1,69)=151.24 p<$ .0001 ; location: $F(1,69)=76.78, p<.0001$; probe location: $F(1,69)=5.27, p=.025$; the interaction between SOA and location: $F(1,69)=7.8, p=.0067$; the interaction between SOA and probe location: $F(1,69)=7.83, p=.0067$; the interaction between location and probe location: $F(1,69)=$ $21.59, p<.0001$; and the interaction between SOA, location, and probe location: $F(1,69)=6.18, p=.0154$.

For the reach-plus-saccade condition, there was a significant effect of the following on performance: SOA: $F(1$, $67)=17.67, p=.0001$; probe location: $F(1,67)=16.88, p$ $=.0001$; the interaction between SOA and location: $F(1$, $67)=9.02, p=.0037$; the interaction between SOA and probe location: $F(1,67)=8.086, p=.0059$; and the interaction between location and probe location: $F(1,67)=$ $7.13, p=.0095$. For the saccade-only condition, there was a significant effect of SOA only on performance, $F(1,69)=42.44, p<.0001$.

A linear mixed-model analysis revealed that there was a significant difference in performance across the time course of the movements between movement conditions (saccade only, reach and saccade, reach only): $F(1,37)=$ $75.89, p<0.0001$; target location $(\mathrm{P} 1, \mathrm{P} 2, \mathrm{O} 1, \mathrm{O} 2): F(1,37)$
$=6.15, p=.018$; and also between probe location relative to movement (in line with or orthogonal): $F(1,37)=$ $12.38, p=.0012$. It can be seen that for the reach and saccade condition in particular, the probe location affects whether there is an overall increase or decrease in performance throughout the movement: for locations in line with the movement direction (P1 and P2), performance decreases throughout the reach, and for locations orthogonal to the movement direction (O1 and $\mathrm{O} 2)$, performance increases throughout the reach. This shows that different types of movement require peak attentional guidance at different points during the movement, and particularly in the case of the reach and saccade condition, the direction of movement additionally affects this.

\section{Performance relative to movement onset}

To compare the performance between the reach-only, saccade-only, and reach-plus-saccade conditions, attentional performance was binned relative to movement onset to see whether the different movement effectors may play a role in the allocation of attention. Figure 4 shows the performance in the two reaching conditions binned relative to either reach onset (a) or saccade onset (b). Note that due to the large bin sizes used $(100 \mathrm{~ms})$, there is still data at the $50 \mathrm{~ms}$ time bin, despite exclusions for saccadic suppression (given the saccadic suppression exclusions were $50 \mathrm{~ms}$ before saccade onset to $25 \mathrm{~ms}$ after saccade onset, and the 0 - 

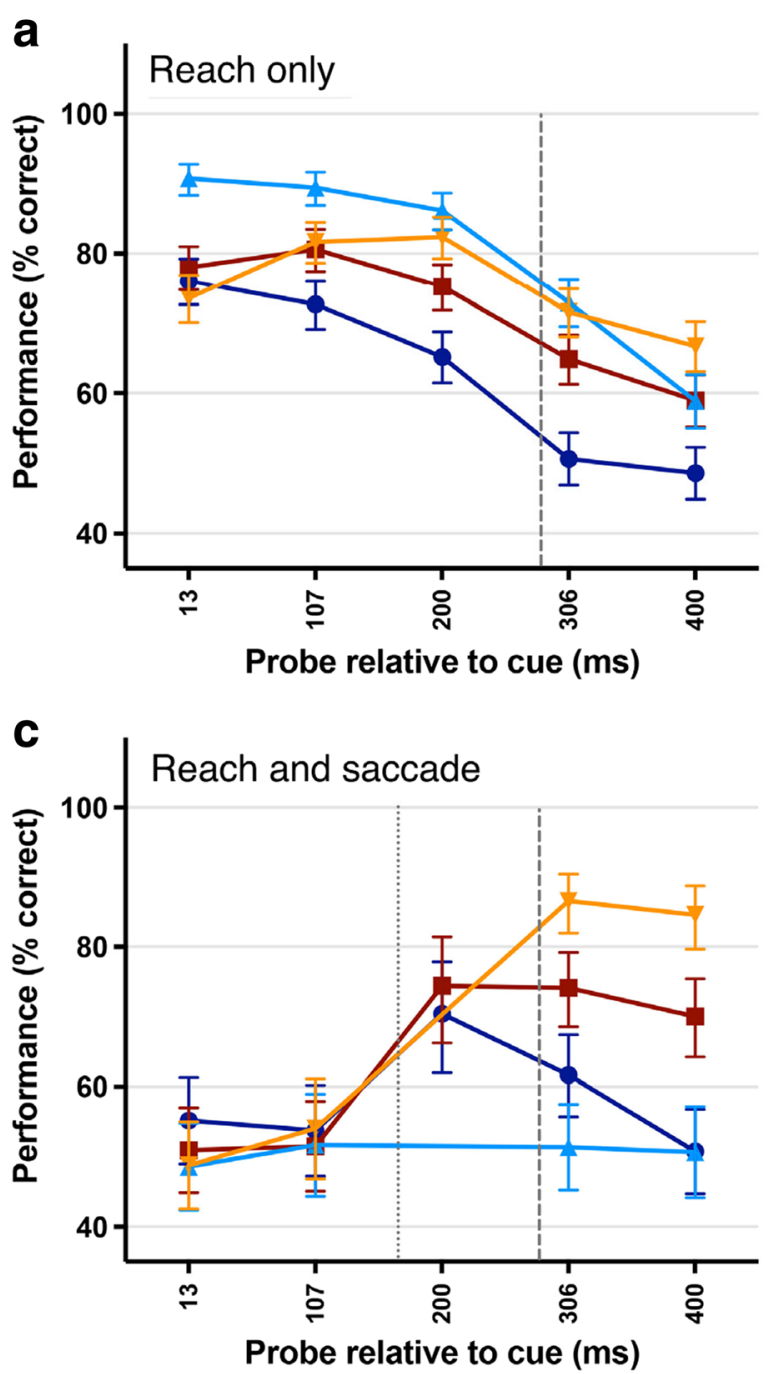

Fig. 3 Performance relative to SOA, grouped by experimental condition. a Performance relative to SOA in the reach-only condition. Dashed vertical line represents median reach latency. b Performance relative to SOA in the saccade-only condition. Dotted vertical line represents median saccade latency. $\mathbf{c}$ Performance relative to SOA in the reach and saccade condition. Error bars for a, b, and $\mathbf{c}$ are Jeffrey's intervals. Dashed

$\mathrm{ms}$ bin should include data from $-50 \mathrm{~ms}$ to $+50 \mathrm{~ms}$, data actually included at the $0-\mathrm{ms}$ bin would be from $25 \mathrm{~ms}$ to $50 \mathrm{~ms}$ after reach onset). Paired-samples $t$ tests were used to quantify the difference in performance before and after movement onset. Data were pooled for each location across the time bins before the movement onset and the time bins after movement onset.

For the reach-plus-saccade condition, when binned relative to reach onset, there was a significant difference for the orthogonal positions: location $\mathrm{O} 1, t(7)=-2.75, p=.028$; location $\mathrm{O} 2, t(7)=-2.9, p=.022$; but no significant difference for the locations in line with the movement direction: location $\mathrm{P} 1$, $t(7)=-0.18, p=.86$; location $\mathrm{P} 2, t(7)=0.34, p=.75$. For the reach-plus-saccade condition, when binned relative to saccade
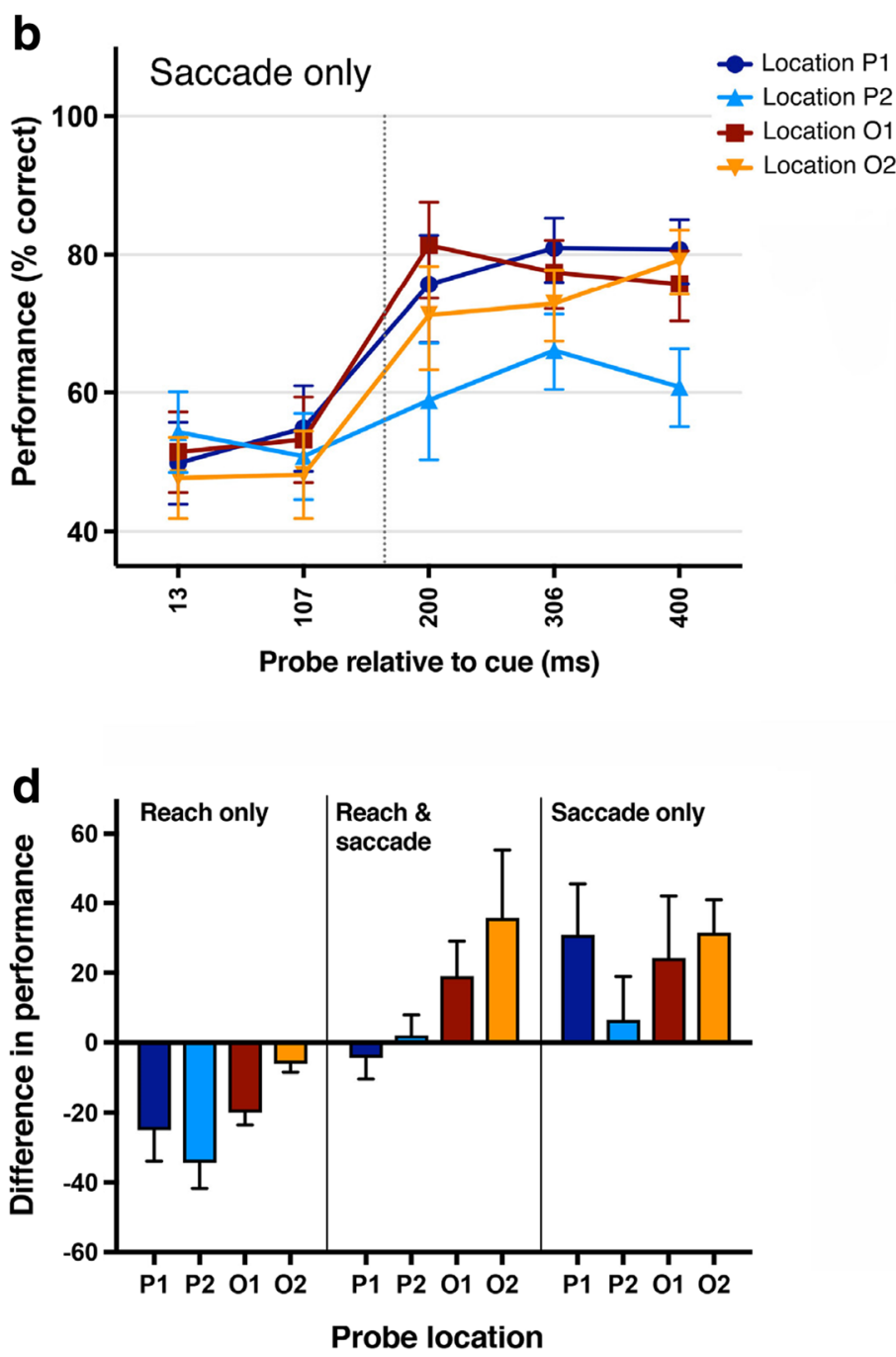

vertical line represents median reach latency and dotted vertical line represents median saccade latency. $\mathbf{d}$ Difference in performance between the shortest SOA and longest SOA for each condition. Blue bars represent parallel probe locations, maroon bars represent orthogonal probe locations. Error bars are $95 \%$ confidence intervals across participant means. (Colour figure online)

onset, there was a significant difference at the orthogonal locations: location $\mathrm{O} 1, t(7)=-2.75, p=.027$; location $\mathrm{O} 2, t(7)=$ $-5.14, p=.013$; however, again there was no significant difference at the locations in line with the movement direction: location P1 $t(7)=-1.3, p=.24$; location $\mathrm{P} 2 t(7)=-0.21, p=$ .84 . For the reach-only condition, when binned relative to reach, there was a significant difference before and after movement onset for all locations: location $\mathrm{P} 1, t(7)=5.69, p$ $=.0007$; location $\mathrm{O} 1, t(7)=4.008, p=.0051 ;$ location $\mathrm{P} 2, t(7)$ $=3.50, p=.01$; location $\mathrm{O} 2, t(7)=3.92, p=.006$. For the saccade-only condition, there was a significant difference at location $\mathrm{P} 1, t(7)=-4.26, p=.0037$; location $\mathrm{O} 1, t(7)=-3.66$, $p=.008$, and location $\mathrm{O} 2, t(7)=-8.80, p=.00005$, but not at location $\mathrm{P} 2, t(7)=-1.64, p=.14$. 


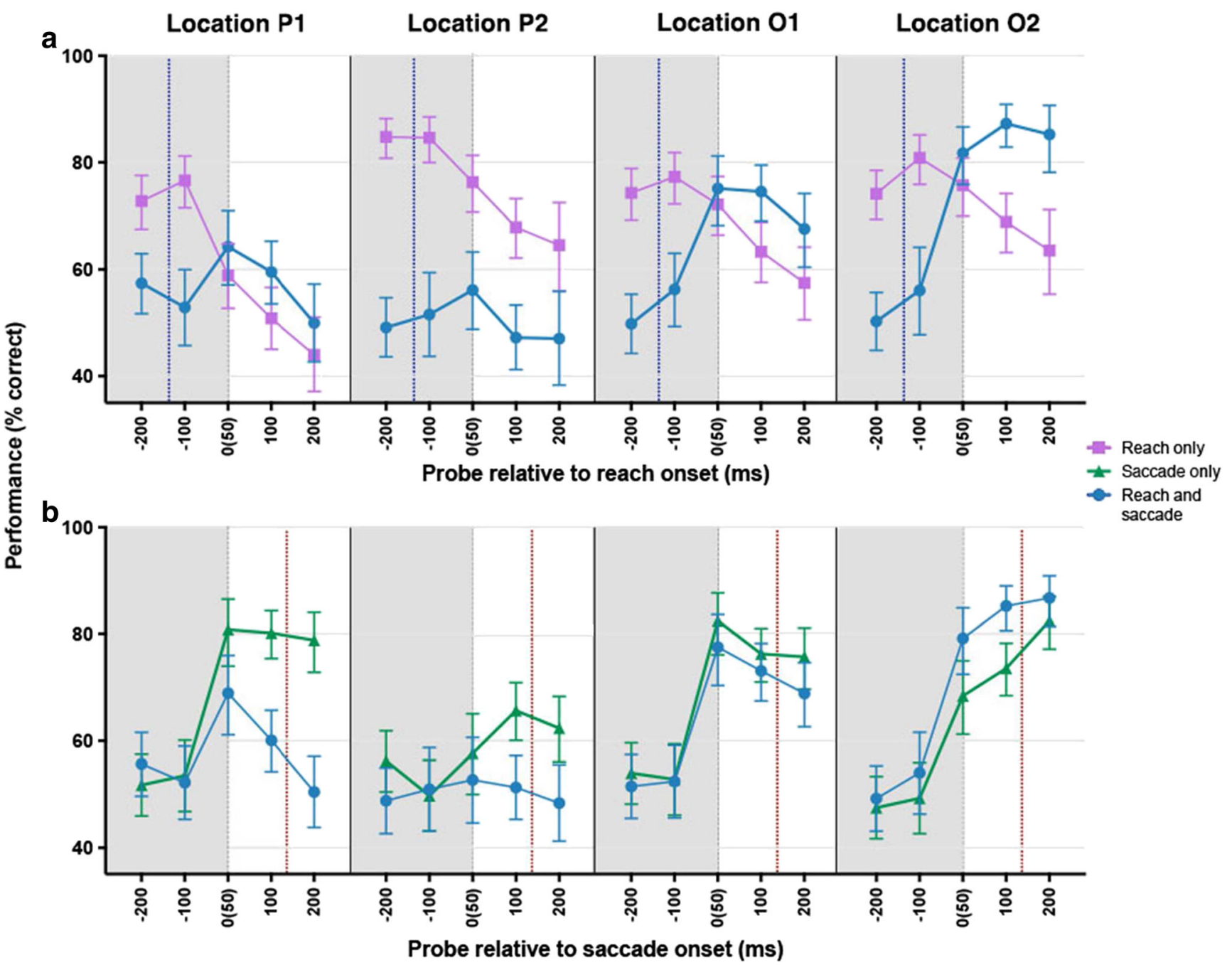

Fig. 4 Performance relative to movement onset for the reach-only, saccade-only, and saccade-plus-reach conditions across all participants. a Performance relative to reach onset for reach-only (purple, squares) and reach-plus-saccade (blue, circles) conditions. Median saccade onset is

\section{Discussion}

The two overarching aims of this experiment were to investigate, first, whether the direction of a movement has an effect on the spatiotemporal characteristics of attention and, second, whether the movement effector affects the time course of attention. It should be noted that the overall aim of the experiment was to map the spatiotemporal profile of attention around the movement target, as facilitation has been found in a number of previous studies at the target location itself (i.e. Deubel, 2008; Deubel \& Schneider, 1996; Jonikaitis \& Deubel, 2011). The overall pattern of results showed that there was a difference in locations in line with or orthogonal to the movement direction, and this differed between the different movement conditions. Results also showed that there was a major difference in the time course of attention, depending on whether an eye movement, hand movement, or both were represented by a dotted blue line. $\mathbf{b}$ Performance relative to saccade onset for saccade-only (green, triangles) and reach-plus-saccade conditions. Error bars are Jeffrey's intervals. Median reach onset is represented by a dotted red line. (Colour figure online)

being enacted. These differences suggest that attention might be differentially and asymmetrically spread around a movement target, depending on the type of movement being made.

\section{Probe location relative to movement affects attentional performance}

When performance is compared across conditions and locations when binned relative to movement onset, it can be seen that for the reach and saccade condition, there is a marked difference in performance at the probe locations in line with and those orthogonal to the movement direction (see Fig. 4a). Comparing this performance to the saccade-only condition when binned relative to saccade onset (see Fig. 4b), it can be seen that performance at the orthogonal locations follows the same general trend between these conditions, while performance at the locations in line with the movement direction 
follows a different pattern between conditions. This suggests that the planning and execution of a reach may differentially affect the profile of attention at parallel and orthogonal locations when that reach is accompanied by a saccade. It also seems to suggest that when a saccade is being made concurrent to a reach, the saccade drives the observed attentional effect, as the profile of attention is markedly different when no saccade is being conducted.

There is little work thus far that has investigated performance at different locations relative to a hand movement in this context; however, these results are consistent with research showing that related movement planning phenomena may show a directional effect in perceptual measures. Perisaccadic mislocalisation has, for example, been shown to have differential effects at locations horizontally in line with and orthogonal to a saccade (Kaiser \& Lappe, 2004), and while this study does not directly test the relationship between aspects of premovement perception such as mislocalisation, attention, and motor control (Hamker, Zirnsak, Calow, \& Lappe, 2008), it may provide a clue as to how premovement attention may fit into this complex system. These findings could, for example, reflect the existence of a neural feedback signal where signals from multiple oculomotor areas send distorting feedback to visual areas, which causes perisaccadic perceptual distortions and may also feed into areas controlling attentional processes (Lappe \& Hamker, 2015). It could be that the same distorted signals causing mislocalisation may also be received by areas involved in attentional selection and processing, and thus the shared asymmetrical properties of presaccadic attention demonstrated in this study may arise from this feedback loop.

This general neural circuit underlying premotor attention shifts may also provide an insight into the premotor planning mechanisms underlying saccade and reach. For example, there is evidence that during the planning and control of reaches, movement guidance and the visual representation of space are closely linked in the early stages of movement planning (e.g. Andersen, Snyder, Bradley, \& Xing, 1997; Ren, 2006). The differentiation of the profile of attention between saccade- and reach-only seen in this study may be indicative of differences in the underlying mechanisms between saccade and reach planning during these early stages of perceptual visual information integration and movement planning: the shift of premotor attention when a saccade is being conducted seems to be a distinct signature of underlying saccade-related mechanisms, which is markedly different from that of a reach alone.

The ways in which the direction of a movement may affect attention is also an important problem to consider in the broader context of completing a reach in a more complex or naturalistic task, where movements may be enacted in a multitude of different directions. This multidimensional attentional problem has been conceptualized in terms of a general priority map that selects targets in a scene and then directs attention to that location (Fecteau \& Munoz, 2006) and that, indeed, attention may form an active "attentional landscape" to select upcoming movement locations (Baldauf \& Deubel, 2010). It is not unreasonable to suggest that in the course of creating such a multidimensional map, the direction of an upcoming movement may be taken into account by attentional selection mechanisms, and attention may be spread in a nonuniform manner to locations in line with or orthogonal to a particular movement target. These ideas about attentional priority maps, target selection, attention, and perisaccadic mislocalisation may seem disparate; however, they ultimately aim to explain how enacting a movement changes perception at the movement target, and how this may affect attentional allocation and the role it plays in the execution of complex motor movements.

\section{Time course of attention is dependent on movement effector}

A comparison of the premovement attention shift between effectors shows that there is a marked difference between saccade-only, saccade-plus-reach, and reach-only conditions in terms of where the peak attentional facilitation is throughout the movement (see Fig. 3). It can be seen that in conditions where a saccade is being made, attention peaks toward the end of the movement, whereas when a reach alone is being conducted, attention decreases across the time course of the movement. These differing temporal profiles may be indicative of which stage in the movement planning and execution process requires the most attentional guidance.

When a reach alone was being enacted, attention peaked toward the start of the movement, degrading during the reach, with lowest attentional performance occurring at the longest SOA (as seen in Fig. 3). When a saccade alone was being completed, the converse trend was observed, as an attentional facilitation was observed over the time course of the movement (see Fig. 3). These patterns seen in the saccade and reach alone conditions are consistent with our previous work with analogous temporal and spatial paradigms, with the reachonly paradigm showing a general degradation across the time course of the reach (Stewart \& Ma-Wyatt, 2015). The saccade condition is also generally consistent with prior research showing this presaccadic attentional shift happens around $200 \mathrm{~ms}$ to $300 \mathrm{~ms}$ after the cue (Castet et al., 2006; Deubel, 2008; Rolfs \& Carrasco, 2012). The condition that incorporated both saccade and reach showed a less consistent time course across locations. As Fig. 3 shows, for the locations orthogonal to the direction of the movements (locations O1 and $\mathrm{O} 2$ ), the observed pattern coheres to the general temporal pattern of premotor attention observed in past research (Jonikaitis \& Deubel, 2011; Rolfs et al., 2013; White et al., 2013). The locations that are in line with the movement direction (locations P1 and P2), however, are less consistent with 
prior empirical evidence, as the performance is relatively flat: indeed, these locations are also somewhat depressed compared to the other conditions. Possible reasons for this suppressed performance will be discussed further in the following sections.

However, when taken together, these results do indicate that the type of movement being made determines whether attention builds up or degrades across the course of that movement. These differences may reflect how attention is utilized during different types of movement planning. For example, for a reach alone, it seems that peak attentional guidance is needed at the outset of the movement planning phase, and once the hand is nearing the target, attention is not so important. This reflects the importance of vision in the planning stage of an accurate hand movement (Ma-Wyatt \& McKee, 2006, 2007). When a movement alone is being planned, there is no saccadic guidance to provide information from the fovea, hence the role of attention in providing higher acuity visual information in its place may be imperative for planning an accurate hand movement.

\section{Suppressed performance: An argument for attentional inhibition}

The saccade-only condition is fairly consistent across both parallel and orthogonal locations, with the exception of location P2, which shows a consistent depression. For the saccade and reach condition, orthogonal locations show a similar pattern to the saccade-only condition, but the locations in line with the movement direction both show a depression in performance. There is no one factor in this experiment which parsimoniously explains why attentional performance should be uniformly worse at these locations when both a saccade and reach are being made, compared to one movement type alone, nor at location $\mathrm{P} 2$ when a saccade alone is being made. There are a number of possible explanations for this pattern: overall, methodological issues that may explain this depressed performance can be dismissed, and it seems more likely that attention is being inhibited at those locations.

The most obvious explanation would be to assume that the hand movement blocked the probe during the course of the reach, hence suppressing performance. This is unlikely, given the high performance in the reach-only condition: if hand interference was the issue, then performance in both reach conditions should be suppressed. Additionally, given the timing of reach latency and reach time, this would be unlikely: the average reach latency was $287 \mathrm{~ms}$ and the average reach time was $222 \mathrm{~ms}$, meaning the total average reach from cue to touch was $509 \mathrm{~ms}$. Given that the last SOA was at $400 \mathrm{~ms}$, it is unlikely that the hand would have been occluding the probe by that time point, and this explanation cannot account for low performance at earlier time points when the hand would not yet be in the vicinity of the target.
Another possible explanation is that individual differences in movement latencies may have eclipsed any attentional effect when the data were collapsed across participants. We investigated this by conducting correlations between reach latency, saccade latency, eye-hand latency, and attentional performance across all participants, at each location and SOA. Results showed that none of these factors were associated with higher or lower performance at these locations (or indeed any locations).

An alternate explanation is that the direction of the saccade had an effect on the perceptual representation of the probes as horizontal and vertical saccades are controlled by different mechanisms; however, this does not seem likely to have caused any effect, as previous studies suggest that perceptual changes such as perisaccadic mislocalisation are no different between horizontal and vertical saccades (Honda, 1989; 1991).

The most parsimonious explanation for this observed depression is that it reflects the dual facilitation/inhibition nature of attention, and this explanation can be used to explain the observed depression in the saccade-only condition, too. There are numerous studies suggesting that attention may actively inhibit stimuli at nonattended areas, or areas surrounding the attended zone: in visual-search tasks, items neighbouring the attended area were found to be suppressed (Bahcall \& Kowler, 1999; Caputo \& Guerra, 1998), and attention has also displayed centre-surround facilitation/inhibition properties in both psychophysical studies (Cutzu \& Tsotsos, 2003) and neurophysiological studies (Hopf et al., 2006; Kim \& Verghese, 2012; Schall, Sato, Thompson, Vaughn, \& Juan, 2004). Additionally, in sequential movement studies, nonrelevant upcoming movement locations were found to be suppressed compared to locations where future movements were to be directed (Baldauf \& Deubel, 2009; Baldauf et al., 2006; Doré-Mazars, Pouget, \& Beauvillain, 2004). It is worth noting that in contrast to some previous studies, isolated targets were used in our paradigm, and only a single movement was required on each trial. It could be the case here that when planning the reach and saccade to the target, locations P1 and P2 become inhibited due to their proximity to the target. It is unclear why this may happen only at these locations, during this one condition, but it could be due to the behavioural irrelevance of the location in comparison to the more important movement target: when conducting a movement toward the target in a direction in line with the target, it may be that the probe at 8 degrees was suppressed in order to enhance facilitation at the 10-degree target.

\section{Conclusion}

This study has shown that the position of a location relative to a movement can affect the temporal profile of attention at that 
location, and this changes between eye and hand movements. These findings indicate that while enacting a movement elicits an attentional shift to an upcoming movement location, the spread of this attentional window is not symmetrical, and making different types of movements in different directions changes when and where attention is allocated.

\section{References}

Andersen, R. A., Snyder, L. H., Bradley, D. C., \& Xing, J. (1997). Multimodal representation of space in the posterior parietal cortex and its use in planning movements. Annual Review of Neuroscience, 20, 303-330. doi:10.1146/annurev.neuro.20.1.303

Bahcall, D. O., \& Kowler, E. (1999). Attentional interference at small spatial separations. Vision Research, 39(1), 71-86.

Baldauf, D., \& Deubel, H. (2009). Attentional selection of multiple goal positions before rapid hand movement sequences: An event-related potential study. Journal of Cognitive Neuroscience, 21(1), 18-29. doi:10.1163/156856897X00249

Baldauf, D., \& Deubel, H. (2010). Attentional landscapes in reaching and grasping. Vision Research, 50(11), 999-1013. doi:10.1016/j.visres. 2010.02 .008

Baldauf, D., Wolf, M., \& Deubel, H. (2006). Deployment of visual attention before sequences of goal-directed hand movements. Vision Research, 46(26), 4355-4374. doi:10.1016/j.visres.2006.08.021

Brainard, D. H. (1997). The psychophysics toolbox. Spatial Vision, 10, 433-436

Caputo, G., \& Guerra, S. (1998). Attentional selection by distractor suppression. Vision Research, 38(5), 669-689.

Castet, E., Jeanjean, S., Montagnini, A., Laugier, D., \& Masson, G. S. (2006). Dynamics of attentional deployment during saccadic programming. Journal of Vision, 6, 3. doi:10.1167/6.3.2

Cutzu, F., \& Tsotsos, J. K. (2003). The selective tuning model of attention: Psychophysical evidence for a suppressive annulus around an attended item. Vision Research, 43(2), 205-219.

Deubel, H. (2008). The time course of presaccadic attention shifts. Psychological Research, 72(6), 630-640. doi:10.1007/s00426008-0165-3

Deubel, H., \& Schneider, W. X. (1996). Saccade target selection and object recognition: Evidence for a common attentional mechanism. Vision Research, 36(12), 1827-1837.

Doré-Mazars, K., Pouget, P., \& Beauvillain, C. (2004). Attentional selection during preparation of eye movements. Psychological Research, 69(1/2), 67-76. doi:10.1007/s00426-003-0166-1

Fecteau, J. H., \& Munoz, D. P. (2006). Salience, relevance, and firing: A priority map for target selection. Trends in Cognitive Sciences, 10(8), 382-390. doi:10.1016/j.tics.2006.06.011

Hamker, F. H., Zirnsak, M., Calow, D., \& Lappe, M. (2008). The perisaccadic perception of objects and space. PLoS Computational Biology, 4(2), e31-15. doi:10.1371/journal.pcbi.0040031

$\mathrm{He}, \mathrm{P}$ and Kowler, E. (1989): The role of location probability in the programming of saccades: Implications for "center-of-gravity" tendencies. Vision Research 29(9): 1165-1181 doi:10.1016/00426989(89)90063-1

Honda, H. (1989). Perceptual localization of visual stimuli flashed during saccades. Perception \& Psychophysics, 45(2), 162-174.

Honda, H. (1991). The time courses of visual mislocalization and of extraretinal eye position signals at the time of vertical saccades. Vision Research, 31(11), 1915-1921.

Hopf, J. M., Boehler, C. N., Luck, S. J., Tsotsos, J. K., Heinze, H. J., \& Schoenfeld, M. A. (2006). Direct neurophysiological evidence for spatial suppression surrounding the focus of attention in vision. Proceedings of the National Academy of Sciences of the United States of America, 103(4), 1053-1058. doi:10.1073/pnas. 0507746103

Jonikaitis, D., \& Deubel, H. (2011). Independent allocation of attention to eye and hand targets in coordinated eye-hand movements. Psychological Science, 22(3), 339-347. doi:10.1177/ 0956797610397666

Kaiser, M., \& Lappe, M. (2004). Perisaccadic mislocalization orthogonal to saccade direction. Neuron, 41(2), 293-300.

Kim, Y. J., \& Verghese, P. (2012). The selectivity of task-dependent attention varies with surrounding context. Journal of Neuroscience, 32(35), 12180-12191. doi:10.1523/JNEUROSCI. 5992-11.2012

Kowler, E., Anderson, E., Dosher, B., \& Blaser, E. (1995). The role of attention in the programming of saccades. Vision Research, 35(13), 1897-1916.

Lappe, M., Awater, H., \& Krekelberg, B. (2000). Postsaccadic visual references generate presaccadic compression of space. Nature, 403(6772), 892-895. doi:10.1038/35002588

Lappe, M. \& Hamker, F. H. (2015). Peri-saccadic compression to two locations in a two-target choice saccade task. Frontiers in Systems Neuroscience. doi:10.3389/fnsys.2015.00135

Legge, G. E., Kersten, D. and Burgess, A. E. (1987). Contrast discrimination in noise. Journal of the Optical Society of America A 4(2), 391-404. doi:10.1364/JOSAA.4.000391

Ma-Wyatt, A., \& McKee, S. P. (2006). Initial visual information determines endpoint precision for rapid pointing. Vision Research, 46(28), 4675-4683. doi:10.1016/j.visres.2006.08.009

Ma-Wyatt, A., \& McKee, S. P. (2007). Visual information throughout a reach determines endpoint precision. Experimental Brain Research, 179(1), 55-64. doi:10.1007/s00221-006-0767-1

Montagnini, A., \& Castet, E. (2007). Spatiotemporal dynamics of visual attention during saccade preparation: Independence and coupling between attention and movement planning. Journal of Vision, 7(14), 8-8. doi:10.1167/7.14.8

Morrone, M. C., Ma-Wyatt, A. and Ross, J. (2005). Seeing and pointing at perisaccadic targets. Journal of Vision 5(9), 741-754 doi:10.1167/ 5.9 .7

Morrone, M. C., Ross, J., \& Burr, D. (2005). Saccadic eye movements cause compression of time as well as space. Nature Neuroscience, 8(7), 950-954. doi:10.1038/nn1488

Pelli, D. (1997). The VideoToolbox software for visual psychophysics: Transforming numbers into movies. Spatial Vision, 10, 437-442

Ren, L. (2006). Proprioceptive guidance of saccades in eye-hand coordination. Journal of Neurophysiology, 96(3), 1464-1477. doi:10. 1152/jn.01012.2005

Rolfs, M., \& Carrasco, M. (2012). Rapid simultaneous enhancement of visual sensitivity and perceived contrast during saccade preparation. Journal of Neuroscience, 32(40), 13744-13752. doi:10.1523/ JNEUROSCI.2676-12.2012

Rolfs, M., Lawrence, B. M., \& Carrasco, M. (2013). Reach preparation enhances visual performance and appearance. Philosophical Transactions of the Royal Society, B: Biological Sciences, 368(1628), 20130057-20130057. doi:10.1167/9.8.30

Schall, J. D., Sato, T. R., Thompson, K. G., Vaughn, A. A., \& Juan, C.-H. (2004). Effects of search efficiency on surround suppression during visual selection in frontal eye field. Journal of Neurophysiology, 91(6), 2765-2769. doi:10.1152/jn.00780.2003

Song, J. H., \& Nakayama, K. (2006). Role of focal attention on latencies and trajectories of visually guided manual pointing. Journal of Vision, 6(9). doi:10.1167/6.9.11

Stewart, E. E. M., \& Ma-Wyatt, A. (2015). The spatiotemporal characteristics of the attentional shift relative to a reach. Journal of Vision, 15(5), 10. doi:10.1167/15.5.10 
Watson, A. B., \& Pelli, D. G. (1983). QUEST: A Bayesian adaptive psychometric method. Perception \& Psychophysics, 33(2), 113120.

White, A. L., Rolfs, M., \& Carrasco, M. (2013). Adaptive deployment of spatial and feature-based attention before saccades. Vision Research, 85(C), 26-35. doi:10.1016/j.visres.2012.10.017
Zhao, M., Gersch, T. M., Schnitzer, B. S., Dosher, B. A., \& Kowler, E. (2012). Eye movements and attention: The role of pre-saccadic shifts of attention in perception, memory and the control of saccades. Vision Research, 74(C), 40-60. doi:10.1016/j.visres.2012.06.017 